\title{
Discontinuity Preserving Method for Noise Removal of Multi-Carrier Signals
}

\author{
Sasan Mahmoodi
}

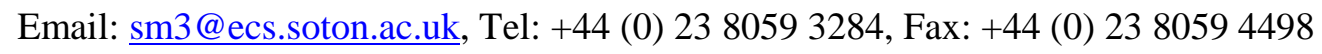

\begin{abstract}
A nonlinear method based on anisotropic diffusion notion is proposed in this paper to remove noise from noisy signals modulated with multiple carrier signals by preserving carrier signals as well as discontinuities present in the original noiseless signals. Gaussian and correlated noise contaminating signals with up to four carriers are considered here. Our algorithm proposed here is implemented with both explicit and semi-implicit discretization schemes. Experiments presented here demonstrate promising results indicating a better performance for our nonlinear noise removal method in comparison with the state-of- art in the literature.
\end{abstract}

Keywords: Anisotropic Diffusion, Noise Removal, Signal Discontinuity, Multi-Carrier Signals, Discontinuity Preserving,

\section{INTRODUCTION}

The significance of the orthogonal frequency-division multiplexing (OFDM) over single-carrier modulation techniques is that OFDM can cope with severe channel conditions such as high frequency attenuation in a long wire, narrowband interference and frequency selective fading because of multipath. In the current state-of-the art, the effect of additive noise such as white Gaussian noise (AWGN) and correlated noise is considered to evaluate some characteristics of the system such as its bit error rate (BER) performance (see e.g. [1] and [2]). No concrete work in the literature exists for noise removal from noisy signals with multiple carriers by preserving some key features of the original noiseless signals such as carrier signals and discontinuities separating the consecutive bits. In this paper, we present a nonlinear filter based on the notion of anisotropic diffusion to remove noise from noisy signals and preserve key features of the original noiseless signals such as carrier signals and discontinuities. The notion of anisotropic diffusion as a nonlinear filter to remove the noise and preserve discontinuities in images is introduced by Perona and Malik in [3]. Such a nonlinear filter is 
generalized for 3D volumetric MRI images in [4] and 2D colour images in [5]. A robust algorithm to estimate a piecewise smooth image from the noisy image is developed by Black et al. in [6]. Tschumperie [7] proposes a fast anisotropic smoothing algorithm based on curvature-preserving partial differential equations (PDE) for the noise removal of multi-valued images. A nonlinear band pass filtering algorithm based on anisotropic diffusion for Binary Phase-Shift Keying (BPSK) signals is proposed by Mahmoodi [8]. This algorithm however can be applied to only complex signals and therefore requires calculating the imaginary part of a real valued signal before the filtering process starts. A nonlinear band pass filtering algorithm based on anisotropic diffusion for real valued signals is therefore proposed in [9] to avoid the requirement for the construction of the imaginary part of a real valued signal. Our contributions in this paper are as follows:

i) The anisotropic diffusion based filtering method for band pass signals is extended for carriers with multiple frequencies. This extension enables us to perform the noise removal for OFDM signals by preserving discontinuities and all carrier signals.

ii) The algorithm proposed here is also implemented by employing a semi-implicit discretization scheme in contrast with the previous works in which only explicit discretization technique is used.

iii) Correlated and white Gaussian noise is considered to demonstrate the efficiency of our proposed filtering method.

There are four major differences between the work presented in this paper and the previous works in [8] and [9]:

a) The noise removal methods in [8] and [9] are for signals with a single carrier frequency; however the nonlinear filter presented here is extended for signals with multiple carrier frequencies. As demonstrated in this paper, such an extension is not trivial. A numerical experiment is also presented in section IV to demonstrate that the noise removal technique for single frequency signals [8] fails for signals with multiple carrier frequencies. 
b) The nonlinear filters in [8] and [9] are numerically implemented by using an explicit discretization scheme; however our system in this paper is implemented by employing a semiimplicit discretization technique to increase the numerical stability of our algorithm here in comparison with the algorithm presented in [8] and [9].

c) The noise removal method discussed in [9] is for real valued signals (with a single carrier frequency) and is a special case of the noise removal algorithm for signals with the double carrier frequencies discussed here. Thus the PDE associated with the noise removal presented in [9] possesses real valued coefficients. However PDEs presented in this paper is more general and have complex valued coefficients.

d) The theoretical foundation for double frequency noise removal methods for signals free from discontinuities (linear case) is also established in this paper. As a result, the propagator for a PDE related to a noise removal system for signals with double carrier frequencies is analytically derived here.

The structure of the rest of the paper is as follows. The theory is outlined in section II. Section III deals with implementation issues. The results are presented in section IV and finally conclusions are drawn in section $\mathrm{V}$.

\section{THEORY}

The anisotropic diffusion equation, used as a nonlinear noise removal method for low pass signals by Perona and Malik [3] is written as.

$$
\left(\frac{\partial}{\partial t}-\left(\frac{\partial}{\partial x}\right) K\left(\frac{\partial}{\partial x}\right)\right) u=0
$$

with initial and boundary conditions:

$$
\begin{aligned}
& u(x, 0)=y(x) \\
& u(0, t)=y(0)
\end{aligned}
$$


$u(L, t)=y(L)$

where $u:\left[\begin{array}{ll}0 & L\end{array}\right] \times R^{+} \rightarrow R, \quad y:\left[\begin{array}{ll}0 & L\end{array}\right] \rightarrow R$ ( $L$ is the length of signal) and $K \geq 0$ are the smoothed low pass signal at the iteration (virtual time) $t$, the original noisy signal and a function of $x$ respectively. The aforementioned partial differential equation is extended by Mahmoodi [8] for the noise removal of band pass signals with a single carrier with a constant frequency $\omega_{1}$ as follows:

$\left(\frac{\partial}{\partial t}-\left(\frac{\partial}{\partial x}-j \omega_{1}\right) K_{1}\left(\frac{\partial}{\partial x}-j \omega_{1}\right)\right) u=0$

with initial and boundary conditions:

$u(x, 0)=y(x)$

$u(0, t)=y(0)$

$u(L, t)=y(L)$

where $u:\left[\begin{array}{ll}0 & L\end{array}\right] \times R^{+} \rightarrow R, y:\left[\begin{array}{ll}0 & L\end{array}\right] \rightarrow R$ and $K_{1} \geq 0$ are the smoothed signal, the noisy signal, and a function of $x$ respectively and also $j=\sqrt{-1}$. Boundary conditions (4-b) and (4-c) are reasonable, because we have no information about the signal at locations $x<0$ or $x>L$. Therefore the values of the smoothed signal at $x=0$ and $x=L$ should remain unchanged. The best guess for the fixed values in these locations is the values of the noisy signal at $x=0$ and $x=L$. Anisotropic diffusion equation (3) with initial and boundary conditions (4) provides a method to smooth band pass signals with a single carrier with frequency $\omega_{1}$.

\section{II-A) NOISE REMOVAL FOR SignALS WITH TwO CARRIER FREQUENCIES}

In this subsection, we propose a higher order anisotropic diffusion equation for the noise removal of noisy signals with two carrier frequencies. Let us assume that the anisotropic diffusion associated with 
noisy signals with carrier frequency $\omega_{1}$ is given by equations (3) and (4) and the anisotropic diffusion equation associated with band bass signals whose carrier frequency is $\omega_{2}$, can be written as:

$\left(\frac{\partial}{\partial t}-\left(\frac{\partial}{\partial x}-j \omega_{2}\right) K_{2}\left(\frac{\partial}{\partial x}-j \omega_{2}\right)\right) u=0$

with initial and boundary conditions:

$u(x, 0)=y(x)$

$u(0, t)=y(0)$

$u(L, t)=y(L)$

To smooth a band pass signal with two carrier frequencies $\omega_{1}$ and $\omega_{2}$ such as an OFDM signal, equations (3) and (5) should be combined. It is noted that any linear combination (i.e. weighted summation/subtraction) of these equations would not be able to smooth a band pass signal with two carrier frequencies. In this paper, we therefore propose the following equation to smooth a band pass signal with carrier frequencies $\omega_{1}$ and $\omega_{2}$ :

$\left\{\frac{\partial}{\partial t}-\left(\frac{\partial}{\partial x}-j \omega_{1}\right) K_{1}\left(\frac{\partial}{\partial x}-j \omega_{1}\right)\right\}\left\{\frac{\partial}{\partial t}-\left(\frac{\partial}{\partial x}-j \omega_{2}\right) K_{2}\left(\frac{\partial}{\partial x}-j \omega_{2}\right)\right\} u=0$

with initial conditions:

$u(x, 0)=y(x)$

$\frac{\partial u(x, 0)}{\partial t}=0$

and boundary conditions:

$u(0, t)=y(0)$ 
$u(L, t)=y(L)$

The rationale behind the idea proposed here as equation (7) for signals with double carrier frequencies is explained in appendix A.

PDE (7) can also be written as:

$$
\begin{aligned}
& \frac{\partial^{2} u}{\partial t^{2}}=\frac{\partial}{\partial t}\left(\left(\frac{\partial}{\partial x}-j \omega_{1}\right) K_{1}\left(\frac{\partial}{\partial x}-j \omega_{1}\right)+\left(\frac{\partial}{\partial x}-j \omega_{2}\right) K_{2}\left(\frac{\partial}{\partial x}-j \omega_{2}\right)\right) u \\
& -\left(\frac{\partial}{\partial x}-j \omega_{1}\right) K_{1}\left(\frac{\partial}{\partial x}-j \omega_{1}\right)\left(\frac{\partial}{\partial x}-j \omega_{2}\right) K_{2}\left(\frac{\partial}{\partial x}-j \omega_{2}\right) u
\end{aligned}
$$

Theorem 1: The propagator of PDE (10) is given by equation (11) for a constant $K=K_{1}=K_{2}$ :

$$
\begin{aligned}
& u(x, t)=\left(\frac{\left(\omega_{1}-3 \omega_{2}\right) \exp \left(j \omega_{1} x\right)-\left(\omega_{2}-3 \omega_{1}\right) \exp \left(j \omega_{2} x\right)}{8\left(\omega_{1}-\omega_{2}\right) \sqrt{\pi K t}}\right) \exp \left(\frac{-x^{2}}{4 K t}\right) \\
& +\frac{j \exp \left(\frac{j\left(\omega_{1}+\omega_{2}\right) x}{2}\right)}{16 \sqrt{\pi K t}\left(\omega_{1}-\omega_{2}\right)} \int_{-\infty}^{x} \exp \left(\frac{-\tau^{2}}{4 K t}\right)\left(\left(\left(\omega_{1}-3 \omega_{2}\right)\left(\omega_{1}+\omega_{2}\right)+4 \omega_{2}^{2}\right) \exp \left(\frac{j\left(\omega_{1}-\omega_{2}\right) \tau}{2}\right)\right. \\
& \left.-\left(\left(\omega_{2}-3 \omega_{1}\right)\left(\omega_{1}+\omega_{2}\right)+4 \omega_{1}^{2}\right) \exp \left(\frac{j\left(\omega_{2}-\omega_{1}\right) \tau}{2}\right)\right) d \tau \\
& +\frac{\exp \left(\frac{-x^{2}}{4 K t}\right)}{4\left(\omega_{1}-\omega_{2}\right) \sqrt{\pi K t}}\left(\frac{j x}{2 K t}\left(\exp \left(j \omega_{1} x\right)-\exp \left(j \omega_{2} x\right)\right)+\left(\omega_{1} \exp \left(j \omega_{1} x\right)-\omega_{2} \exp \left(j \omega_{2} x\right)\right)\right)
\end{aligned}
$$

The proof is presented in Appendix B.

It is important to note that PDE (10) is used to remove noise from complex valued band pass signals. For PDE (10), if there is a discontinuity in the carrier signal with frequency $\omega_{1}$, then there will be a 
peak in $\left(\frac{\partial u}{\partial x}-j \omega_{1} u\right)$ at the point of discontinuity. Therefore $K_{1}$ should be a function of $x$ and needs to approach zero at such a discontinuity [8]. In such a case, $u$ at this discontinuity point remains unchanged with respect to $t$. Hence the discontinuity in the carrier signal with frequency $\omega_{1}$ is preserved. Similarly, if there is a discontinuity at a point in the carrier signal with frequency $\omega_{2}$, then $K_{2}$ being a function of $x$, should approach zero to preserve such a discontinuity at that point. This aforementioned notion is employed in this paper to numerically implement anisotropic diffusion algorithm to smooth noisy signals with two carrier frequencies. The numerical implementation is described in more details in section III. If $\omega_{1}=\omega_{0}$ and $\omega_{2}=-\omega_{0}$, then $\operatorname{PDE}(10)$ can be exploited for the noise removal of real valued band pass signals with carrier frequency of $\omega_{0}$ [9]. In a linear case where $K=K_{1}=K_{2}$ is a constant, $\mathrm{PDE}(10)$ is simplified to the following equation:

$$
\frac{\partial^{2} u}{\partial t^{2}}=K \frac{\partial}{\partial t}\left(\left(\frac{\partial}{\partial x}-j \omega_{0}\right)^{2}+\left(\frac{\partial}{\partial x}+j \omega_{0}\right)^{2}\right) u-K^{2}\left(\frac{\partial}{\partial x}-j \omega_{0}\right)^{2}\left(\frac{\partial}{\partial x}+j \omega_{0}\right)^{2} u
$$

Or

$\frac{\partial^{2} u}{\partial t^{2}}=2 K \frac{\partial}{\partial t}\left(\frac{\partial^{2}}{\partial x^{2}}-\omega_{0}^{2}\right) u-K^{2}\left(\frac{\partial}{\partial x}+\omega_{0}^{2}\right)^{2} u$

PDE (12) is a differential equation with real valued coefficients and therefore its solution is also a real valued signal $u$. According to theorem 1, the propagator of PDE (12) can then be calculated as:

$$
\begin{aligned}
& u(x, t)=\left(\frac{\cos \left(\omega_{0} x\right)}{2 \sqrt{\pi K t}}\right) \exp \left(\frac{-x^{2}}{4 K t}\right)-\frac{\omega_{0}}{4 \sqrt{\pi K t}} \int_{-\infty}^{x} \exp \left(\frac{-\tau^{2}}{4 K t}\right) \sin \left(\omega_{0} \tau\right) d \tau \\
& +\left(\frac{-x \sin \left(\omega_{0} x\right)+\omega_{0} \cos \left(\omega_{0} x\right)}{4 \omega_{0} \sqrt{\pi K t}}\right)\left(\exp \left(\frac{-x^{2}}{4 K t}\right)\right)
\end{aligned}
$$


PDEs (7) and (10) can be generalized to smooth noisy signals with three carrier frequencies as follows:

$$
\begin{aligned}
& \left(\frac{\partial}{\partial t}-\left(\frac{\partial}{\partial x}-j \omega_{1}\right) K_{1}\left(\frac{\partial}{\partial x}-j \omega_{1}\right)\right)\left(\frac{\partial}{\partial t}-\left(\frac{\partial}{\partial x}-j \omega_{2}\right) K_{2}\left(\frac{\partial}{\partial x}-j \omega_{2}\right)\right) \times \ldots \\
& \times\left(\frac{\partial}{\partial t}-\left(\frac{\partial}{\partial x}-j \omega_{3}\right) K_{3}\left(\frac{\partial}{\partial x}-j \omega_{3}\right)\right) u=0
\end{aligned}
$$

with initial conditions:

$u(x, 0)=y(x)$

$\frac{\partial u(x, 0)}{\partial t}=0$

$\frac{\partial^{2} u(x, 0)}{\partial t^{2}}=0$

and boundary conditions:

$u(0, t)=y(0)$

$u(L, t)=y(L)$

where $\omega_{1}, \omega_{2}$ and $\omega_{3}$ are the carrier frequencies of the noisy signal $u(x, 0)$. PDE (14) can also be rewritten as:

$\frac{\partial^{3} u}{\partial t^{3}}=\left[\frac{\partial^{2}}{\partial t^{2}}\left(a_{1}+a_{2}+a_{3}\right)-\frac{\partial}{\partial t}\left(a_{1} a_{2}+a_{1} a_{3}+a_{2} a_{3}\right)+a_{1} a_{2} a_{3}\right] u$

where $\omega_{i} \mathrm{~s}(i=1,2$ and 3$)$ are the frequencies of the carrier signals and 
$a_{i}=\left(\frac{\partial}{\partial x}-j \omega_{i}\right) K_{i}\left(\frac{\partial}{\partial x}-j \omega_{i}\right)$

$i=1,2$ and 3

Similar to PDE(10), in PDE (17), $K_{i}$ s, (i= 1, 2 and 3) being functions of $x$, need to approach zero at the points of discontinuities. A numerical method in section III is described to ensure that $K_{i} \mathrm{~s}$, approach zero at discontinuities in carrier signals with frequencies $\omega_{i} \mathrm{~s}(i=1,2$ and 3$)$.

The extension of PDE (17) to signals with four carrier frequencies is straightforward as follows:

$$
\begin{aligned}
& \left(\frac{\partial}{\partial t}-\left(\frac{\partial}{\partial x}-j \omega_{1}\right) K_{1}\left(\frac{\partial}{\partial x}-j \omega_{1}\right)\right)\left(\frac{\partial}{\partial t}-\left(\frac{\partial}{\partial x}-j \omega_{2}\right) K_{2}\left(\frac{\partial}{\partial x}-j \omega_{2}\right)\right) \\
& \times\left(\frac{\partial}{\partial t}-\left(\frac{\partial}{\partial x}-j \omega_{3}\right) K_{3}\left(\frac{\partial}{\partial x}-j \omega_{3}\right)\right)\left(\frac{\partial}{\partial t}-\left(\frac{\partial}{\partial x}-j \omega_{4}\right) K_{4}\left(\frac{\partial}{\partial x}-j \omega_{4}\right)\right) u=0
\end{aligned}
$$

PDE (18) can then be re-written as:

$$
\begin{aligned}
& \frac{\partial^{4} u}{\partial t^{4}}=\left[\frac{\partial^{3}}{\partial t^{3}}\left(a_{1}+a_{2}+a_{3}+a_{4}\right)-\frac{\partial^{2}}{\partial t^{2}}\left(a_{1} a_{2}+a_{1} a_{3}+a_{1} a_{4}+a_{2} a_{3}+a_{2} a_{4}+a_{3} a_{4}\right)+\right. \\
& \left.+\frac{\partial}{\partial t}\left(a_{1} a_{2} a_{3}+a_{1} a_{2} a_{4}+a_{2} a_{3} a_{4}+a_{1} a_{3} a_{4}\right)+a_{1} a_{2} a_{3} a_{4}\right] u
\end{aligned}
$$

where $\omega_{i} \mathrm{~s}(i=1,2,3$ and 4$)$ are the frequencies of the carrier signals and

$$
a_{i}=\left(\frac{\partial}{\partial x}-j \omega_{i}\right) K_{i}\left(\frac{\partial}{\partial x}-j \omega_{i}\right)
$$

Similar to the method described for PDEs (10) and (18), $K_{i}$ s, (i=1,2, 3 and 4) being functions of $x$, should approach zero at the points of discontinuities. Extension for the signals with the higher number of carrier frequencies than four is also straightforward. The general form of an anisotropic diffusion 
equation for noisy signals containing $n$ carrier signals to remove the noise and to preserve discontinuities as well as carrier signals is as follows:

$$
\left[\prod_{i=1}^{n}\left(\frac{\partial}{\partial t}-a_{i}\right)\right] u=0
$$

where

$$
a_{i}=\left(\frac{\partial}{\partial x}-j \omega_{i}\right) K_{i}\left(\frac{\partial}{\partial x}-j \omega_{i}\right) \quad i=1,2, \ldots, n
$$

\section{DISCRETIZATION AND IMPLEMENTATION}

Perona and Malik in their seminal work [3] employ an explicit discretization scheme to implement their anisotropic diffusion equation for image noise removal. Explicit discretization methods are also used to implement anisotropic diffusion equation for band pass signals with a single carrier frequency [8][9]. The main advantage of explicit discretization methods is that it is easy to implement. However since anisotropic diffusion equations are stiff, the algorithm is unstable, unless the step size $\Delta t$ is taken very small. However the smaller, the step size, the slower the algorithm approaches to the desired solution. The discretization scheme used in this paper is semi implicit method which is stable for higher values of step sizes. As a result, the algorithm implemented by the semi-implicit discretization scheme converges to the desired solution faster when it is implemented by the explicit discretization scheme. The discretization method for signals with two carrier frequencies based on PDE (10) is presented in subsection (III-A). In subsection (III-B), the semi implicit discretization for PDEs containing multiple carrier frequencies are also discussed. The Euclidean distance between the smoothed signals in two consecutive iterations is used as a stopping condition. When such a distance is less than a user defined threshold $\left(T_{s}\right)$, the algorithm stops. Obviously the lower the threshold $T_{s}$ is chosen, the smoother the signal and the longer the running time of our algorithm will be. Parameter $\Delta x$ is set to unity in the all experiments in this paper. Parameter $\Delta t$ plays an important role in the stability and the speed of convergence of algorithm. Lower values for $\Delta t$ cause the algorithm to 
approach to the desired solution more slowly. Higher values for $\Delta t$, on the other hand, increases the running speed of the algorithm if there is no instability. But the algorithm may also become unstable with such high values of $\Delta t$. Parameter $q$ is chosen based on the amount of transitions in discontinuities of the original noiseless signal and the level of noise in the noisy signal. For signals contaminated with severe noise, larger value of $q$, would be more convenient. To avoid smoothing discontinuities in less noisy signals, it is best to choose lower values for $q$. Our assumption here is that the original noiseless signal has at least one full cycle of the carrier signals between two consecutive discontinuities to avoid difficulties associated with the uncertainty principle [10].

\section{III-A) DISCRETIZATION FOR SIGNALS WITH TWO CARRIER FREQUENCIES}

PDE (10) can be discretized explicitly and implicitly. However an implicit method is employed in this paper to discretize PDE (10). Since PDE (10) is generally a nonlinear equation, we can therefore exploit a semi implicit scheme for discretization. Equation (10) can be discretized as follows:

$$
\begin{aligned}
& u(x, t)=2 u(x, t-\Delta t)-u(x, t-2 \Delta t) \\
& \left.+\Delta t \mid K_{L}^{\omega_{1}}(u) D_{\omega_{1}}^{L}(u(x, t))+K_{R}^{\omega_{1}}(u) D_{\omega_{1}}^{R}(u(x, t))-j \omega_{1} K_{R}^{\omega_{1}}(u) D_{\omega_{1}}^{R}(u(x, t))\right] \\
& \left.-\Delta t \mid K_{L}^{\omega_{1}}(u) D_{\omega_{1}}^{L}(u(x, t-\Delta t))+K_{R}^{\omega_{1}}(u) D_{\omega_{1}}^{R}(u(x, t-\Delta t))-j \omega_{1} K_{R}^{\omega_{1}}(u) D_{\omega_{1}}^{R}(u(x, t-\Delta t))\right] \\
& \left.+\Delta t \mid K_{L}^{\omega_{2}}(u) D_{\omega_{2}}^{L}(u(x, t))+K_{R}^{\omega_{2}}(u) D_{\omega_{2}}^{R}(u(x, t))-j \omega_{2} K_{R}^{\omega_{2}}(u) D_{\omega_{2}}^{R}(u(x, t))\right] \\
& \left.-\Delta t \mid K_{L}^{\omega_{2}}(u) D_{\omega_{2}}^{L}(u(x, t-\Delta t))+K_{R}^{\omega_{2}}(u) D_{\omega_{2}}^{R}(u(x, t-\Delta t))-j \omega_{2} K_{R}^{\omega_{2}}(u) D_{\omega_{2}}^{R}(u(x, t-\Delta t))\right] \\
& -\Delta t^{2}\left\{\mid K_{L}^{\omega_{1}}(w) D_{\omega_{1}}^{L}(w(x, t))+K_{R}^{\omega_{1}}(w) D_{\omega_{1}}^{R}(w(x, t))-j \omega_{1} K_{R}^{\omega_{1}}(w) D_{\omega_{1}}^{R}(w(x, t))\right] \\
& \left.\times\left[K_{L}^{\omega_{2}}(u) D_{\omega_{2}}^{L}(u(x, t))+K_{R}^{\omega_{2}}(u) D_{\omega_{2}}^{R}(u(x, t))-j \omega_{2} K_{R}^{\omega_{2}}(u) D_{\omega_{2}}^{R}(u(x, t))\right]\right\}
\end{aligned}
$$

For $x=1,2,3, \ldots, N$

where $N$ is the length of the original noisy signal and

$$
w(x, t)=K_{L}^{\omega_{2}}(u) D_{\omega_{2}}^{L}(u(x, t))+K_{R}^{\omega_{2}}(u) D_{\omega_{2}}^{R}(u(x, t))-j \omega_{2} K_{R}^{\omega_{2}}(u) D_{\omega_{2}}^{R}(u(x, t)),
$$


And also

$$
\begin{array}{ll}
D_{\omega_{1}}^{L}(w(x, t))=\frac{w(x-\Delta x, t)-w(x, t)}{\Delta x}+j \omega_{1} w(x, t), & \\
D_{\omega_{1}}^{R}(w(x, t))=\frac{w(x+\Delta x, t)-w(x, t)}{\Delta x}-j \omega_{1} w(x, t), & \\
K_{L}^{\omega_{1}}(w)=\frac{1}{\Delta x} g\left(\left|D_{\omega_{1}}^{L}(w(x, t-\Delta t))\right|\right) \text { and } K_{R}^{\omega_{1}}(w)=\frac{1}{\Delta x} g\left(\left|D_{\omega_{1}}^{R}\left(w_{R}(x, t-\Delta t)\right)\right|\right), & \text { for } i=1,2 \\
D_{\omega_{1}}^{L}(u(x, t))=\frac{u(x-\Delta x, t)-u(x, t)}{\Delta x}+j \omega_{i} u(x, t), & \text { for } i=1,2 \\
D_{\omega_{1}}^{R}(u(x, t))=\frac{u(x+\Delta x, t)-u(x, t)}{\Delta x}-j \omega_{i} u(x, t), & \\
K_{L}^{\omega_{i}}(u)=\frac{1}{\Delta x} g\left(\left|D_{\omega_{i}}^{L}(u(x, t-\Delta t))\right|\right) \text { and } K_{R}^{\omega_{i}}\left(u_{R}\right)=\frac{1}{\Delta x} g\left(\left|D_{\omega_{i}}^{R}(u(x, t-\Delta t))\right|\right), & \text { for } i=1,2
\end{array}
$$

Function $g(y)$ is set to one of the following functions:

$$
g(y)=\exp \left(-\frac{y^{2}}{q^{2}}\right)
$$

Or

$$
g(y)=\frac{1}{1+\frac{y^{2}}{q^{2}}}
$$

where $q$ is a parameter set by the user.

In equations (20), there are terms such as $K_{L}^{\omega_{1}}(u)$ and $K_{R}^{\omega_{1}}(u)$ that are nonlinearly related to the values of $u(x, t)$. These terms are considered as implicit terms and to avoid a set of nonlinear equations, these terms are estimated at iteration $t-\Delta t$ and therefore are treated as coefficients. On 
the other hand, the terms that are linearly related to $u(x, t)$ are regarded as explicit and therefore are considered as unknowns. In such a scheme, equations (20) therefore becomes a linear system in which the unknowns are $u(x, t)$ for various values of $x$. It is more convenient to write equations (20) in matrix form. To this end, let us write equations (20) with respect to $u(x-\Delta x, t), u(x, t)$ and $u(x+\Delta x, t)$, i.e.:

$A u(x-\Delta x, t)+B u(x, t)+C u(x+\Delta x, t)=D$

where the coefficients $A, B, C$, and $D$ are evaluated in the previous iteration i.e., at $t-\Delta t$ and therefore they are considered as known coefficients in the current iteration. These coefficients are calculated as follows:

$$
\begin{aligned}
& A(x)=-\frac{\Delta t}{\Delta x} K_{L}^{\omega_{1}}(u)-\frac{\Delta t}{\Delta x} K_{L}^{\omega_{2}}(u) \\
& B(x)=1+\frac{\Delta t}{\Delta x} K_{R}^{\omega_{1}}(u)+\frac{\Delta t}{\Delta x} K_{L}^{\omega_{1}}(u)-j \Delta t \omega_{1} K_{L}^{\omega_{1}}(u)-j \frac{\Delta t}{\Delta x} \omega_{1} K_{R}^{\omega_{1}}(u)+\frac{\Delta t}{\Delta x} K_{L}^{\omega_{2}}(u) \\
& -j \Delta t \omega_{2} K_{L}^{\omega_{2}}(u)+\frac{\Delta t}{\Delta x} K_{R}^{\omega_{2}}(u)-j \frac{\Delta t}{\Delta x} \omega_{2} K_{R}^{\omega_{2}}(u) \\
& C(x)=-\frac{\Delta t}{\Delta x} K_{R}^{\omega_{1}}(u)+j \Delta t \omega_{1} K_{R}^{\omega_{1}}(u)+j \frac{\Delta t}{\Delta x} \omega_{1} K_{R}^{\omega_{1}}(u)+\Delta t \omega_{1}^{2} K_{R}^{\omega_{1}}(u)-\frac{\Delta t}{\Delta x} K_{R}^{\omega_{2}}(u)+j \Delta t \omega_{2} K_{R}^{\omega_{2}}(u) \\
& +j \frac{\Delta t}{\Delta x} \omega_{2} K_{R}^{\omega_{2}}(u)+\Delta t \omega_{2}^{2} K_{R}^{\omega_{2}}(u)
\end{aligned}
$$

$$
\begin{aligned}
& D(x)=2 u(x, t-\Delta t)-u(x, t-2 \Delta t)-\Delta t\left\{K_{L}^{\omega_{1}}(u) D_{\omega_{1}}^{L}(u(x, t-\Delta t))+K_{R}^{\omega_{1}}(u) D_{\omega_{1}}^{R}(u(x, t-\Delta t))\right. \\
& \left.-j \omega_{1} K_{R}^{\omega_{1}}(u) D_{\omega_{1}}^{R}(u(x, t-\Delta t))\right\}-\Delta t\left\{K_{L}^{\omega_{2}}(u) D_{\omega_{2}}^{L}(u(x, t-\Delta t))+K_{R}^{\omega_{2}}(u) D_{\omega_{2}}^{R}(u(x, t-\Delta t))\right. \\
& \left.-j \omega_{1} K_{R}^{\omega_{1}}(u) D_{\omega_{1}}^{R}(u(x, t-\Delta t))\right\}-\Delta t^{2}\left\{K_{L}^{\omega_{1}}(w) D_{\omega_{1}}^{L}(w(x, t-\Delta t))+K_{R}^{\omega_{1}}(w) D_{\omega_{1}}^{R}(w(x, t-\Delta t))\right. \\
& \left.-j \omega_{1} K_{R}^{\omega_{1}}(w) D_{\omega_{1}}^{R}(w(x, t-\Delta t))\right\}
\end{aligned}
$$

Linear system (24) is therefore written in a matrix form as follows: 
$\left[\begin{array}{cccccccc}1 & 0 & 0 & 0 & 0 & 0 & \ldots & 0 \\ A(2) & B(2) & C(2) & 0 & 0 & 0 & \ldots & 0 \\ 0 & A(3) & B(3) & C(3) & 0 & 0 & \ldots & 0 \\ 0 & 0 & A(4) & B(4) & C(4) & 0 & \ldots & 0 \\ \ldots & \ldots & \ldots & \ldots & \ldots & \ldots & \ldots & \ldots \\ 0 & \ldots & 0 & 0 & A(n-2) & B(n-2) & C(n-2) & 0 \\ 0 & \ldots & 0 & 0 & 0 & A(n-1) & B(n-1) & C(n-1) \\ 0 & \ldots & 0 & 0 & 0 & 0 & 0 & 1\end{array}\right]\left[\begin{array}{c}u(1, t) \\ u(2, t) \\ u(3, t) \\ u(4, t) \\ \ldots \\ u(n-2, t) \\ u(n-1, t) \\ u(n, t)\end{array}\right]=\left[\begin{array}{c}u(1,0) \\ D(2) \\ D(3) \\ D(4) \\ \cdots \\ D(n-2) \\ D(n-1) \\ u(n, 0)\end{array}\right]$

It is noted that boundary conditions (9-a) and (9-b) are employed in (29). These boundary conditions are reasonable because there is no information about the signal for $x<1$ and $x>n$. If the square $n \times n$ matrix in (29) is full rank, the inverse of this square matrix multiplied by the column vector in the left hand side of (29) is the solution (the smoothed signal at iteration $t$ ). However if this square matrix is a low rank matrix, the pseudo-inverse of this matrix is multiplied by the vector column in the left hand side of (29) to find the solution. The solution of linear system (29) is the smoothed signal $u(x, t)$ in a vector form at iteration $t$. We also notice that linear system (29) is solved for $t \geq 1$. For $t=0$, the signal $u$ is the initial condition $u(x, 0)$.

\section{III-B) DISCRETIZATION FOR SIGNALS WITH MULTIPLE CARRIER FREQUENCIES}

Both explicit and semi-implicit discretization schemes can be used to discretize PDE (17) for signals with three carrier frequencies. In a semi-implicit discretization framework, PDE (17) is discretized as follows:

$$
\begin{aligned}
& u(x, t)=3 u(x, t-\Delta t)-3 u(x, t-2 \Delta t)+u(x, t-3 \Delta t) \\
& \left.+\Delta t \mid K_{L}^{\omega_{1}}(u) D_{\omega_{1}}^{L}(u(x, t))+K_{R}^{\omega_{1}}(u) D_{\omega_{1}}^{R}(u(x, t))-j \omega_{1} K_{R}^{\omega_{1}}(u) D_{\omega_{1}}^{R}(u(x, t))\right] \\
& \left.-2 \Delta t \mid K_{L}^{\omega_{1}}(u) D_{\omega_{1}}^{L}(u(x, t-\Delta t))+K_{R}^{\omega_{1}}(u) D_{\omega_{1}}^{R}(u(x, t-\Delta t))-j \omega_{1} K_{R}^{\omega_{1}}(u) D_{\omega_{1}}^{R}(u(x, t-\Delta t))\right] \\
& +\Delta t\left[K_{L}^{\omega_{1}}(u) D_{\omega_{1}}^{L}(u(x, t-2 \Delta t))+K_{R}^{\omega_{1}}(u) D_{\omega_{1}}^{R}(u(x, t-2 \Delta t))-j \omega_{1} K_{R}^{\omega_{1}}(u) D_{\omega_{1}}^{R}(u(x, t-2 \Delta t))\right] \\
& +\Delta t\left[K_{L}^{\omega_{2}}(u) D_{\omega_{2}}^{R}(u(x, t))+K_{R}^{\omega_{2}}(u) D_{\omega_{2}}^{R}(u(x, t))-j \omega_{2} K_{R}^{\omega_{2}}(u) D_{\omega_{2}}^{R}(u(x, t))\right]
\end{aligned}
$$




$$
\begin{aligned}
& \left.-2 \Delta t \mid K_{L}^{\omega_{2}}(u) D_{\omega_{2}}^{L}(u(x, t-\Delta t))+K_{R}^{\omega_{2}}(u) D_{\omega_{2}}^{R}(u(x, t-\Delta t))-j \omega_{2} K_{R}^{\omega_{2}}(u) D_{\omega_{2}}^{R}(u(x, t-\Delta t))\right] \\
& \left.+\Delta t \mid K_{L}^{\omega_{2}}(u) D_{\omega_{2}}^{L}(u(x, t-2 \Delta t))+K_{R}^{\omega_{2}}(u) D_{\omega_{2}}^{R}(u(x, t-2 \Delta t))-j \omega_{2} K_{R}^{\omega_{2}}(u) D_{\omega_{2}}^{R}(u(x, t-2 \Delta t))\right] \\
& \left.+\Delta t \mid K_{L}^{\omega_{3}}(u) D_{\omega_{3}}^{L}(u(x, t))+K_{R}^{\omega_{3}}(u) D_{\omega_{3}}^{R}(u(x, t))-j \omega_{3} K_{R}^{\omega_{3}}(u) D_{\omega_{3}}^{R}(u(x, t))\right] \\
& -2 \Delta t\left[K_{L}^{\omega_{3}}(u) D_{\omega_{3}}^{L}(u(x, t-\Delta t))+K_{R}^{\omega_{3}}(u) D_{\omega_{3}}^{R}(u(x, t-\Delta t))-j \omega_{3} K_{R}^{\omega_{3}}(u) D_{\omega_{3}}^{R}(u(x, t-\Delta t))\right] \\
& +\Delta t\left[K_{L}^{\omega_{3}}(u) D_{\omega_{3}}^{L}(u(x, t-2 \Delta t))+K_{R}^{\omega_{3}}(u) D_{\omega_{3}}^{R}(u(x, t-2 \Delta t))-j \omega_{3} K_{R}^{\omega_{3}}(u) D_{\omega_{3}}^{R}(u(x, t-2 \Delta t))\right] \\
& \left.-\Delta t^{2} \mid K_{L}^{\prime \omega_{1}}\left(W^{\omega_{2}}\right) D_{\omega_{1}}^{L}\left(W^{\omega_{2}}(x, t-\Delta t)\right)+K_{R}^{\prime \omega_{1}}\left(W^{\omega_{2}}\right) D_{\omega_{1}}^{R}\left(W^{\omega_{2}}(x, t-\Delta t)\right)-j \omega_{1} K_{R}^{\prime \omega_{1}}\left(W^{\omega_{2}}\right) D_{\omega_{1}}^{R}\left(W^{\omega_{2}}(x, t-\Delta t)\right)\right] \\
& +\Delta t^{2}\left\lfloor K_{L}^{\prime \omega_{1}}\left(W^{\omega_{2}}\right) D_{\omega_{1}}^{L}\left(W^{\omega_{2}}(x, t-2 \Delta t)+K_{R}^{\prime \omega_{1}}\left(W^{\omega_{2}}\right) D_{\omega_{1}}^{R}\left(W^{\omega_{2}}(x, t-2 \Delta t)\right)-j \omega_{1} K_{R}^{\prime \omega_{1}}\left(W^{\omega_{2}}\right) D_{\omega_{1}}^{R}\left(W^{\omega_{2}}(x, t-2 \Delta t)\right)\right]\right. \\
& -\Delta t^{2}\left[K_{L}^{\prime \omega_{1}}\left(W^{\omega_{3}}\right) D_{\omega_{1}}^{L}\left(W^{\omega_{3}}(x, t-\Delta t)\right)+K_{R}^{\prime \omega_{1}}\left(W^{\omega_{3}}\right) D_{\omega_{1}}^{R}\left(W^{\omega_{3}}(x, t-\Delta t)\right)-j \omega_{1} K_{R}^{\prime \omega_{1}}\left(W^{\omega_{3}}\right) D_{\omega_{1}}^{R}\left(W^{\omega_{3}}(x, t-\Delta t)\right)\right] \\
& +\Delta t^{2}\left[K_{L}^{\prime \omega_{1}}\left(W^{\omega_{3}}\right) D_{\omega_{1}}^{L}\left(W^{\omega_{3}}(x, t-2 \Delta t)\right)+K_{R}^{\prime \omega_{1}}\left(W^{\omega_{3}}\right) D_{\omega_{1}}^{R}\left(W^{\omega_{3}}(x, t-2 \Delta t)\right)-j \omega_{1} K_{R}^{\prime \omega_{1}}\left(W^{\omega_{3}}\right) D_{\omega_{1}}^{R}\left(W^{\omega_{3}}(x, t-2 \Delta t)\right)\right] \\
& -\Delta t^{2}\left[K_{L}^{\prime \omega_{2}}\left(W^{\omega_{3}}\right) D_{\omega_{2}}^{L}\left(W^{\omega_{3}}(x, t-\Delta t)\right)+K_{R}^{\prime \omega_{2}}\left(W^{\omega_{3}}\right) D_{\omega_{2}}^{R}\left(W^{\omega_{3}}(x, t-\Delta t)\right)-j \omega_{2} K_{R}^{\prime \omega_{2}}\left(W^{\omega_{3}}\right) D_{\omega_{2}}^{R}\left(W^{\omega_{3}}(x, t-\Delta t)\right)\right] \\
& +\Delta t^{2}\left[K_{L}^{\prime \omega_{2}}\left(W^{\omega_{3}}\right) D_{\omega_{2}}^{L}\left(W^{\omega_{3}}(x, t-2 \Delta t)\right)+K_{R}^{\prime \omega_{2}}\left(W^{\omega_{3}}\right) D_{\omega_{2}}^{R}\left(W^{\omega_{3}}(x, t-2 \Delta t)\right)-j \omega_{2} K_{R}^{\prime \omega_{2}}\left(W^{\omega_{3}}\right) D_{\omega_{2}}^{R}\left(W^{\omega_{3}}(x, t-2 \Delta t)\right)\right] \\
& +\Delta t^{3}\left[K_{L}^{\prime \prime \omega_{1}}\left(X^{\omega_{2}, \omega_{3}}\right)\left(D_{\omega_{1}}^{L}\left(X^{\omega_{2}, \omega_{3}}(x, t-\Delta t)\right)+K_{R}^{\prime \prime \omega_{1}}\left(X^{\omega_{2}, \omega_{3}}\right) D_{\omega_{1}}^{R}\left(X^{\omega_{2}, \omega_{3}}(x, t-\Delta t)\right)-j \omega_{1} K_{R}^{\prime \prime \omega_{1}}\left(X^{\omega_{2}, \omega_{3}}\right) D_{\omega_{1}}^{R}\left(X_{R}^{\omega_{2}, \omega_{3}}(x, t-\Delta t)\right)\right]\right.
\end{aligned}
$$

where $x=1,2,3, \ldots, N$ and $N$ is again the length of original noisy signal and

$$
\begin{aligned}
& D_{\omega_{i}}^{L}(u(x, t))=\frac{u(x-\Delta x, t)-u(x, t)}{\Delta x}+j \omega_{i} u(x, t), \\
& D_{\omega_{i}}^{R}(u(x, t))=\frac{u(x+\Delta x, t)-u(x, t)}{\Delta x}-j \omega_{i} u(x, t),
\end{aligned}
$$

$K_{L}^{\omega_{i}}(u)=\frac{1}{\Delta x} g\left(\left|D_{\omega_{i}}^{L}(u(x, t-\Delta t))\right|\right)$ and $K_{R}^{\omega_{i}}(u)=\frac{1}{\Delta x} g\left(\left|D_{\omega_{i}}^{R}(u(x, t-\Delta t))\right|\right), \quad$ for $i=1,2,3$

$W^{\omega_{i}}(x, t)=K_{L}^{\omega_{i}}(u) D_{\omega_{i}}^{L}(u(x, t))+K_{R}^{\omega_{i}}(u) D_{\omega_{i}}^{R}(u(x, t))-j \omega_{i} K_{R}^{\omega_{i}}(u) D_{\omega_{i}}^{R}(u(x, t))$ 


$$
\begin{aligned}
& D_{\omega_{i}}^{L}\left(W^{\omega_{i}}(x, t-\Delta t)\right)=\frac{W^{\omega_{i}}(x-\Delta x, t-\Delta t)-W^{\omega_{i}}(x, t-\Delta t)}{\Delta x}+j \omega_{i} W^{\omega_{i}}(x, t-\Delta t) \text { for } i=1,2,3 \\
& D_{\omega_{i}}^{R}\left(W^{\omega_{i}}(x, t-\Delta t)\right)=\frac{W^{\omega_{i}}(x+\Delta x, t-\Delta t)-W^{\omega_{i}}(x, t-\Delta t)}{\Delta x}-j \omega_{i} W^{\omega_{i}}(x, t-\Delta t) \text { for } i=1,2,3 \\
& K_{L}^{\prime \omega_{i}}\left(W^{\omega_{i}}\right)=\frac{1}{\Delta x} g\left(\left|D_{\omega_{i}}^{L}\left(W^{\omega_{i}}(x, t-\Delta t)\right)\right|\right) \text { and } K_{R}^{\prime \omega_{1}}\left(W^{\omega_{i}}\right)=\frac{1}{\Delta x} g\left(\left|D_{\omega_{i}}^{R}\left(W^{\omega_{i}}(x, t-\Delta t)\right)\right|\right), \text { for } i=1,2,3 \\
& X^{\omega_{2}, \omega_{3}}(x, t-\Delta t)=K_{L}^{\prime \omega_{2}}\left(W^{\omega_{3}}\right) D_{\omega_{2}}^{L}\left(W^{\omega_{3}}(x, t-\Delta t)\right)+K_{R}^{\prime \omega_{2}}\left(W^{\omega_{3}}\right) D_{\omega_{2}}^{R}\left(W^{\omega_{3}}(x, t-\Delta t)\right) \\
& -j \omega_{2} K_{R}^{\prime \omega_{2}}\left(W^{\omega_{3}}\right) D_{\omega_{2}}^{R}\left(W^{\omega_{3}}(x, t-\Delta t)\right) \\
& K_{L}^{\prime \prime \omega_{1}}\left(W^{\omega_{1}}\right)=\frac{1}{\Delta x} g\left(\left|D_{\omega_{1}}^{L}\left(W^{\omega_{1}}(x, t-\Delta t)\right)\right|\right) \text { and } K_{R}^{\prime \prime \omega_{1}}\left(W^{\omega_{1}}\right)=\frac{1}{\Delta x} g\left(\left|D_{\omega_{1}}^{R}\left(W^{\omega_{1}}(x, t-\Delta t)\right)\right|\right) \\
& K_{L}^{\prime \prime \omega_{1}}\left(X^{\omega_{2}, \omega_{3}}\right)=\frac{1}{\Delta x} g\left(\left|D_{\omega_{1}}^{L}\left(X^{\omega_{2}, \omega_{3}}(x, t-\Delta t)\right)\right|\right) \text { and } K_{R}^{\prime \prime \omega_{1}}\left(X^{\omega_{2}, \omega_{3}}\right)=\frac{1}{\Delta x} g\left(\left|D_{\omega_{1}}^{R}\left(X^{\omega_{2}, \omega_{3}}(x, t-\Delta t)\right)\right|\right)
\end{aligned}
$$

Function $g(y)$ is given in equation (22) or (23). Similar to subsection (III-A), nonlinear terms with respect to $u(x, t)$ in (30) are considered as implicit terms and therefore are evaluated at previous iteration and used as coefficients in the current iteration. The terms related to $u(x, t)$ linearly are regarded as explicit terms and are therefore treated as unknowns. As a result, equation (30) can be written as the following linear system, i.e.:

$$
A u(x-\Delta x, t)+B u(x, t)+C u(x+\Delta x, t)=D
$$

where $A, B, C$, and $D$ are coefficients containing terms evaluated at the previous iteration, i.e.:

$$
\begin{aligned}
& A(x)=-\frac{\Delta t}{\Delta x} K_{L}^{\omega_{1}}(u)-\frac{\Delta t}{\Delta x} K_{L}^{\omega_{2}}(u)-\frac{\Delta t}{\Delta x} K_{L}^{\omega_{3}}(u) \\
& B(x)=1+\frac{\Delta t}{\Delta x} K_{L}^{\omega_{1}}(u)-j \Delta t \omega_{1} K_{L}^{\omega_{1}}(u)+\frac{\Delta t}{\Delta x} K_{R}^{\omega_{1}}(u)-j \frac{\Delta t}{\Delta x} \omega_{1} K_{R}^{\omega_{1}}(u)+\frac{\Delta t}{\Delta x} K_{L}^{\omega_{2}}(u)
\end{aligned}
$$




$$
\begin{aligned}
& -j \Delta t \omega_{2} K_{L}^{\omega_{2}}(u)+\frac{\Delta t}{\Delta x} K_{R}^{\omega_{2}}(u)-j \frac{\Delta t}{\Delta x} \omega_{2} K_{R}^{\omega_{2}}(u)+\frac{\Delta t}{\Delta x} K_{L}^{\omega_{3}}(u)-j \Delta t \omega_{3} K_{L}^{\omega_{3}}(u)+\frac{\Delta t}{\Delta x} K_{R}^{\omega_{3}}(u) \\
& -j \frac{\Delta t}{\Delta x} \omega_{3} K_{R}^{\omega_{3}}(u)
\end{aligned}
$$

$$
\begin{aligned}
& C(x)=-\frac{\Delta t}{\Delta x} K_{R}^{\omega_{1}}(u)+j \Delta t \omega_{1} K_{R}^{\omega_{1}}(u)+j \frac{\Delta t}{\Delta x} \omega_{1} K_{R}^{\omega_{1}}(u)+\Delta t \omega_{1}^{2} K_{R}^{\omega_{1}}(u)-\frac{\Delta t}{\Delta x} K_{R}^{\omega_{2}}(u)+j \Delta t \omega_{2} K_{R}^{\omega_{2}}(u) \\
& j \frac{\Delta t}{\Delta x} \omega_{2} K_{R}^{\omega_{2}}(u)+\Delta t \omega_{2}^{2} K_{R}^{\omega_{2}}(u)-\frac{\Delta t}{\Delta x} K_{R}^{\omega_{3}}(u)+j \Delta t \omega_{3} K_{R}^{\omega_{3}}(u)+j \frac{\Delta t}{\Delta x} \omega_{3} K_{R}^{\omega_{3}}(u)+\Delta t \omega_{3}^{2} K_{R}^{\omega_{3}}(u)
\end{aligned}
$$

$$
\begin{aligned}
& D(x)=3 u(x, t-\Delta t)-3 u(x, t-2 \Delta t)+u(x, t-3 \Delta t) \\
& -2 \Delta t\left(K_{L}^{\omega_{1}}(u) D_{\omega_{1}}^{L}(u(x, t-\Delta t))+K_{R}^{\omega_{1}}(u) D_{\omega_{1}}^{R}(u(x, t-\Delta t))-j \omega_{1} K_{R}^{\omega_{1}}(u) D_{\omega_{1}}^{R}(u(x, t-\Delta t))\right) \\
& \left.+\Delta t \mid K_{L}^{\omega_{1}}(u) D_{\omega_{1}}^{L}(u(x, t-2 \Delta t))+K_{R}^{\omega_{1}}(u) D_{\omega_{1}}^{R}(u(x, t-2 \Delta t))-j \omega_{1} K_{R}^{\omega_{1}}(u) D_{\omega_{1}}^{R}(u(x, t-2 \Delta t))\right] \\
& \left.-2 \Delta t \mid K_{L}^{\omega_{2}}(u) D_{\omega_{2}}^{L}(u(x, t-\Delta t))+K_{R}^{\omega_{2}}(u) D_{\omega_{2}}^{R}(u(x, t-\Delta t))-j \omega_{2} K_{R}^{\omega_{2}}(u) D_{\omega_{2}}^{R}(u(x, t-\Delta t))\right] \\
& \left.+\Delta t \mid K_{L}^{\omega_{2}}(u) D_{\omega_{2}}^{L}(u(x, t-2 \Delta t))+K_{R}^{\omega_{2}}(u) D_{\omega_{2}}^{R}(u(x, t-2 \Delta t))-j \omega_{2} K_{R}^{\omega_{2}}(u) D_{\omega_{2}}^{R}(u(x, t-2 \Delta t))\right] \\
& -2 \Delta t\left[K_{L}^{\omega_{3}}(u) D_{\omega_{3}}^{L}(u(x, t-\Delta t))+K_{R}^{\omega_{3}}(u) D_{\omega_{3}}^{R}(u(x, t-\Delta t))-j \omega_{3} K_{R}^{\omega_{3}}(u) D_{\omega_{3}}^{R}(u(x, t-\Delta t))\right] \\
& \left.+\Delta t \mid K_{L}^{\omega_{3}}(u) D_{\omega_{3}}^{L}(u(x, t-2 \Delta t))+K_{R}^{\omega_{3}}(u) D_{\omega_{3}}^{R}(u(x, t-2 \Delta t))-j \omega_{3} K_{R}^{\omega_{3}}(u) D_{\omega_{3}}^{L}(u(x, t-2 \Delta t))\right] \\
& \left.-\Delta t^{2} \mid K_{L}^{\prime \omega_{1}}\left(W^{\omega_{2}}\right) D_{\omega_{1}}^{L}\left(W^{\omega_{2}}(x, t-\Delta t)\right)+K_{R}^{\prime \omega_{1}}\left(W^{\omega_{2}}\right) D_{\omega_{1}}^{R}\left(W^{\omega_{2}}(x, t-\Delta t)\right)-j \omega_{1} K_{R}^{\prime \omega_{1}}\left(W^{\omega_{2}}\right) D_{\omega_{1}}^{R}\left(W^{\omega_{2}}(x, t-\Delta t)\right)\right]
\end{aligned}
$$$$
+\Delta t^{2}\left[K_{L}^{\prime \omega_{1}}\left(W^{\omega_{2}}\right) D_{\omega_{1}}^{L}\left(W^{\omega_{2}}(x, t-2 \Delta t)+K_{R}^{\prime \omega_{1}}\left(W^{\omega_{2}}\right) D_{\omega_{1}}^{R}\left(W^{\omega_{2}}(x, t-2 \Delta t)\right)-j \omega_{1} K_{R}^{\prime \omega_{1}}\left(W^{\omega_{2}}\right) D_{\omega_{1}}^{R}\left(W^{\omega_{2}}(x, t-2 \Delta t)\right)\right]\right.
$$$$
-\Delta t^{2}\left[K_{L}^{\prime \omega_{1}}\left(W^{\omega_{3}}\right) D_{\omega_{1}}^{L}\left(W^{\omega_{3}}(x, t-\Delta t)\right)+K_{R}^{\prime \omega_{1}}\left(W^{\omega_{3}}\right) D_{\omega_{1}}^{R}\left(W_{R}^{\omega_{3}}(x, t-\Delta t)\right)-j \omega_{1} K_{R}^{\prime \omega_{1}}\left(W^{\omega_{3}}\right) D_{\omega_{1}}^{R}\left(W^{\omega_{3}}(x, t-\Delta t)\right)\right]
$$$$
+\Delta t^{2}\left[K_{L}^{\prime \omega_{1}}\left(W^{\omega_{3}}\right) D_{\omega_{1}}^{L}\left(W^{\omega_{3}}(x, t-2 \Delta t)\right)+K_{R}^{\prime \omega_{1}}\left(W^{\omega_{3}}\right) D_{\omega_{1}}^{R}\left(W^{\omega_{3}}(x, t-2 \Delta t)\right)-j \omega_{1} K_{R}^{\prime \omega_{1}}\left(W^{\omega_{3}}\right) D_{\omega_{1}}^{R}\left(W^{\omega_{3}}(x, t-2 \Delta t)\right)\right]
$$$$
-\Delta t^{2}\left[K_{L}^{\prime \omega_{2}}\left(W^{\omega_{3}}\right) D_{\omega_{2}}^{L}\left(W^{\omega_{3}}(x, t-\Delta t)\right)+K_{R}^{\prime \omega_{2}}\left(W^{\omega_{3}}\right) D_{\omega_{2}}^{R}\left(W^{\omega_{3}}(x, t-\Delta t)\right)-j \omega_{2} K_{R}^{\prime \omega_{2}}\left(W^{\omega_{3}}\right) D_{\omega_{2}}^{R}\left(W^{\omega_{3}}(x, t-\Delta t)\right)\right]
$$ 


$$
\begin{aligned}
& +\Delta t^{2}\left[K_{L}^{\prime \omega_{2}}\left(W^{\omega_{3}}\right) D_{\omega_{2}}^{L}\left(W^{\omega_{3}}(x, t-2 \Delta t)\right)+K_{R}^{\prime \omega_{2}}\left(W^{\omega_{3}}\right) D_{\omega_{2}}^{R}\left(W^{\omega_{3}}(x, t-2 \Delta t)\right)-j \omega_{2} K_{R}^{\prime \omega_{2}}\left(W^{\omega_{3}}\right) D_{\omega_{2}}^{R}\left(W^{\omega_{3}}(x, t-2 \Delta t)\right)\right] \\
& +\Delta t^{3}\left[K_{L}^{\prime \prime \omega_{1}}\left(X^{\omega_{2}, \omega_{3}}\right)\left(D_{\omega_{1}}^{L}\left(X^{\omega_{2}, \omega_{3}}(x, t-\Delta t)\right)+K_{R}^{\prime \prime \omega_{1}}\left(X^{\omega_{2}, \omega_{3}}\right) D_{\omega_{1}}^{R}\left(X^{\omega_{2}, \omega_{3}}(x, t-\Delta t)\right)-j \omega_{1} K_{R}^{\prime \prime \omega_{1}}\left(X^{\omega_{2}, \omega_{3}}\right) D_{\omega_{1}}^{R}\left(X^{\omega_{2}, \omega_{3}}(x, t-\Delta t)\right)\right]\right.
\end{aligned}
$$

Therefore linear system (31) can be written in the matrix form of equation (29) with the same boundary conditions corresponding to boundary conditions (16-a) and (16-b).

Semi-implicit discretization for signals with four carrier frequencies is also similar to those for signals with two and three carrier frequencies presented in the previous and the current subsections and therefore are skipped here.

\section{RESULTS}

The first example presented here deals with a signal with no discontinuity. Figure (1-a) shows a signal with two carrier signals with frequencies $\omega_{1}=0.1, \omega_{2}=0.2 \mathrm{rad} / \mathrm{s}$. White zero mean Gaussian noise is added to this signal to produce the noisy signal with $\mathrm{SNR}_{\mathrm{dB}}=-0.82$, as depicted in figure (1-b). Since there is no discontinuity in the original noiseless signal, then the noise reduction can be achieved by convolving the noisy signal with propagator (11). Since the operation is linear (convolution), therefore it is not iterative, and no time step setup is required for this experiment. The smoothed signal is presented in figure (1-c).

In the next example, white Gaussian noise is added to a typical OFDM signal (shown in figure (2-a)) containing three discontinuities and modulated with two carrier signals with frequencies 0.25 and 0.45 $\mathrm{rad} / \mathrm{s}$ to produce a noisy signal with $\mathrm{SNR}_{\mathrm{dB}}=-0.19$, as shown in figure (2-b). The double frequency anisotropic diffusion discussed in subsection (II-A) with $q=10$ and $\Delta t=0.01$ is applied to the noisy signal of figure (2-b) to produce the smoothed signal shown in figure (2-c). As shown from this figure, the noise is removed and the discontinuities and carrier signals are preserved. The single carrier anisotropic diffusion algorithm [8] with frequency $0.25 \mathrm{rad} / \mathrm{s}$ is also applied to the noisy signal of figure (2-b) to produce the smoothed signal shown in figure (2-d) for comparison purposes. As can 
be seen from figure (2-d), only one carrier signal is preserved in the smoothed signal and all discontinuities are smoothed. As can be seen from this figure, the discontinuities located at 100, 200 and 300 in the horizontal axis are preserved in the smoothed (filtered) signal shown in figure (2-c). However by increasing the noise level (or lowering the SNR) in the noisy signal, the discontinuities in the smoothed (filtered) signal may be smoothed or some discontinuities associated with the noise and not the original noiseless signal, may be preserved in the smoothed signal as reported in [11].

The next experiment compares the explicit and implicit methods for the discretization of PDE (10). An explicit method similar to one discussed in [8] for the discretization of PDE (10) with parameters $\Delta t=0.0005, q=10$ is applied to the noisy signal of figure (2-b) to produce the signal smoothed after 1000 and 4000 iterations, as shown in figures (3-a) and (3-b) respectively. As can be seen from figure (3), the signal smoothed after 1000 iterations is still noisy. The signal obtained after 4000 iterations and shown in figure (3-b) is more smoothed but it is not as smoothed as the signal smoothed by the semi implicit method (see figure (2-c)) taking only 600 iterations. Semi-implicit discretization techniques are well known to bring more numerical stability to the numerical solutions of the nonlinear differential equations. However this more numerical stability may come with more numerical costs for CPU times. The time costs for both algorithms to reach a certain numerical accuracy for solutions may vary, and primarily depend on the value of $\Delta t$. The possible highest value set for $\Delta t$ also depends on the amount of noise present in the signal, as noise is one of the parameters which can cause instability in the algorithm. Figure (4-a) shows a typical OFDM noiseless signal modulated with two carrier signals with frequencies $\omega_{1}=0.2, \omega_{2}=0.5 \mathrm{rad} / \mathrm{s}$.

This signal is contaminated with a correlated Gaussian noise (white Gaussian noise filtered with the transfer function $\left.H(z)=\frac{1}{\sum_{n=1}^{10} z^{-n+1}}\right)$ to produce a noisy signal with $S N R_{d B}=5.44$, as depicted in figure (4-b). The nonlinear noise removal algorithm proposed in section II-A for band pass signals modulated with two carrier signals is applied to this noisy signal to produce the smoothed signal shown in figure (4-c). As observed from this figure, the signal is smoothed. But the original 
discontinuities of the noiseless signals as well as both carrier signals are preserved in the smoothed signal. Figure (5) shows that in the next experiment our algorithm smooths a noisy signal modulated with carrier signals with three different frequencies $\omega_{1}=0.0625, \omega_{2}=0.125$ and $\omega_{3}=0.25 \mathrm{rad} / \mathrm{s}$. White Gaussian noise is added to the noiseless signal with three aforementioned carrier frequencies shown in figure (5-a) to produce the noisy signal shown in figure (5-b) with $S N R_{d B}=12.04$.

Our anisotropic diffusion technique for three carrier signals explained in section II-B is applied to this noisy signal to produce the smoothed signal shown in figure (5-c). As shown from this figure, signal discontinuities as well as carrier signals are preserved.

Figure (6-a) also shows a typical OFDM signal modulated with four carrier signals $\left(\omega_{1}=0.0313\right.$, $\omega_{2}=0.0625, \omega_{3}=0.125$ and $\left.\omega_{4}=0.25 \mathrm{rad} / \mathrm{s}\right)$. This noiseless signal is contaminated with white Gaussian noise to produce the noisy signal of figure (6-b) with $S N R_{d B}=8.92$. Our anisotropic diffusion algorithm for band pass OFDM signals with four carrier frequencies is applied to the noisy signal of figure (6-b) to produce the smoothed signal shown in figure (6-c). As observed from this figure, original signal discontinuities as well as carrier signals are persevered in the smoothed signal.

Let us now see how the performance of the algorithm implemented with the semi-implicit scheme changes under the variations of the algorithm's parameters as well as under the presence of noise. Our anisotropic diffusion implemented with the semi-implicit scheme with double carrier frequencies with $q=10$, and $\Delta t=0.01$ is applied to a noisy OFDM signal with two carrier frequencies $\omega_{1}=0.125$, $\omega_{2}=0.5 \mathrm{rad} / \mathrm{s}$ which contains discontinuities and is contaminated with Gaussian noise with $S N R_{d B}=4.38$. In this experiment, we measure an error term (equation (36)) as a difference between the original noiseless signal and the smoothed signal filtered by the algorithm applied to the noisy signal as follows:

Error $=\frac{\sum_{n=1}^{N}\left|u(n)-u_{f}(n)\right|^{2}}{N}$ 
where $u(n)$ and $u_{f}(n)$ are noiseless and filtered discrete signals respectively with total number of $N$ samples. This error term represents the performance of the algorithm. Less the value of the error term, the better the performance of the algorithm is. The error term measured in this experiment is plotted in figure (7) against the convergence time of the algorithm implemented with the semi-implicit method discussed in section III-A. As can be seen in this figure, lower values for error results in more computation time. After a point where the computation (convergence) time is around 10 seconds, the error decreases very slowly. A slow decrease in error term after this point causes the computation (convergence) time to increase significantly. Figure (8) shows how the error term (the algorithm performance) measured in (36) and convergence time for the anisotropic diffusion algorithm implemented with semi-implicit scheme with double carrier frequencies, changes by varying parameter $q$ and keeping the other parameters of the experiment constant ( $\Delta t=0.01, \omega_{1}=0.125$, $\omega_{2}=0.5, S N R_{d B}=4.38$ ). As can be seen from this figure, the convergence (computation) time to process the signal remains almost unchanged with various values for parameter $q$ as shown in figure (8-b). However the error term (measured in equation (36)) is minimal for a certain value of $q$ as shown in figure (8-a). In this experiment, the value of $q$ for which the error is minimal is around 10. By increasing the value of parameter $q$, the error slightly increases. This is due to the fact that higher values for $q$ cause slightly over-smoothing in the signal. Values of $q$ lower than 10 also increase the error term significantly, because more noise remains in the filtered signal for lower values of $q$. Figure (9) shows how the error and convergence time change with respect to $\Delta t$ for $q=10$ and $S N R_{d B}=4.38$. As can be seen from figure (9-a), the error representing the performance of the algorithm remains unchanged by varying $\Delta t$. On the other hand, the convergence time decreases as $\Delta t$ increases as shown in figure (9-b). However for the values of $\Delta t$ greater than 0.02 , the algorithm becomes unstable and it therefore diverges. The error and convergence time of the anisotropic algorithm with double carrier frequencies $\left(\omega_{1}=0.125, \omega_{2}=0.5\right)$ for $\Delta t=0.01$ and $q=10$ with respect to SNR of the noisy signal are depicted in figures (10-a) and (10-b) respectively. As shown in figure (10-a), if noise level increases in the signal (i.e. SNR decreases), the error (measured by using 
equation (36)) also increases. This is expected, because more level of noise increases the error in the filtered signal. On the other hand, the convergence time also increases as the noise level increases. This is also predictable, because the algorithm needs more iterations and therefore more time to smooth the noisy signal, if the noise level becomes higher. In the final experiment, zero mean Gaussian noise is added to the signal shown in figure (2-a) to produce a noisy signal with $S N R=0.77_{\mathrm{dB}}$. Our anisotropic diffusion algorithm for double frequencies (with frequencies 0.25 and 0.45 ) implemented with the semi-implicit discretization method for $q=10$ and $\Delta t=0.01$, is applied to this noisy signal for noise removal. The algorithm converges after 300 iterations. Figure (11) shows the evolution of SNR (i.e. the SNR of the smoothed signal in each iteration) with respect to the iteration number. As can be seen from this figure, the SNR of the smoothed signal increases as our algorithm approaches the convergence. As observed from this figure, the SNR of the original noisy signal is $0.77_{\mathrm{dB}}$ and the SNR of the smoothed signal in the final iteration increases to $15.13_{\mathrm{dB}}$. It is noted that all experiments performed in this paper are implemented in Matlab version R2015b run on a PC with dual core processors each with $2.60 \mathrm{GHz}$ frequency.

\section{CONCLUSION}

A nonlinear noise removal method based on anisotropic diffusion for OFDM signals modulated with multiple carrier signals is proposed in this paper. Such a nonlinear filtering technique reduces the noise from noisy OFDM signals and preserves discontinuities as well as the carrier signals. A semi implicit discretization technique is employed here to improve the smoothing property and stability of our nonlinear noise removal algorithm. Our algorithm implemented by semi-implicit and explicit discretization schemes are compared in this paper. Various levels of white and correlated Gaussian noise are considered here to demonstrate the ability of our algorithm for noise removal. The performance of our nonlinear noise reduction algorithm is evaluated here at the presence of various noise levels and also with various values for the algorithm's parameters. The results presented in this paper indicate that our nonlinear noise reduction algorithm is robust in severe noisy environments. 


\section{APPENDICES}

\section{Appendix A:}

\section{Justification for Anisotropic Equation with Multiple Carrier Frequencies:}

In this appendix, we would like to explain the inspiration for the nonlinear noise removal method we propose in this paper for signals with multiple carrier signals. Let us start with signals with double frequency carriers. The generalization to more than two frequencies is then straightforward. As discussed in [8], the spatial temporal impulse response (propagator) in Fourier and Laplace domain for PDE (3) with carrier frequency $\omega_{1}$ is as follows:

$$
P_{1}(\omega, s)=\frac{1}{s+K_{1}\left(\omega-\omega_{1}\right)^{2}}
$$

where $s$ and $\omega$ are temporal complex frequency (s-plane in Laplace domain) and spatial frequency (Fourier domain) respectively. Similarly, the spatial temporal impulse response (propagator) in Fourier and Laplace domain for PDE (5) with carrier frequency $\omega_{2}$ can be written as:

$$
P_{2}(\omega, s)=\frac{1}{s+K_{2}\left(\omega-\omega_{2}\right)^{2}}
$$

Let us now assume that the desirable PDE can be written as:

$$
D\left(\omega_{1}, \omega_{2}\right)(u)=0
$$

where $D\left(\omega_{1}, \omega_{2}\right)$ is a linear differential operator consisting of spatial and temporal derivatives and a function of two carrier frequencies $\omega_{1}$ and $\omega_{2}$. Let us further assume that the spatial temporal impulse response (propagator) for PDE (A-3) is a linear combination of propagators (A-1) and (A-2). The spatial temporal impulse response (propagator) for PDE (A-3) in space-time domain can therefore be written as:

$u(x, t)=A p_{1}\left(x, t ; \omega_{1}\right)+B p_{2}\left(x, t ; \omega_{2}\right)$ 
where $p_{1}$ and $p_{2}$ are the spatial temporal impulse responses of PDEs (3) and (5) in space-time domain and A and B are constant. As a result, impulse response (A-4) can be written in FourierLaplace domain as:

$U(\omega, s)=\frac{A}{s+K_{1}\left(\omega-\omega_{1}\right)^{2}}+\frac{B}{s+K_{2}\left(\omega-\omega_{2}\right)^{2}}$

Or

$\left(s+K_{1}\left(\omega-\omega_{1}\right)^{2}\right)\left(s+K_{2}\left(\omega-\omega_{2}\right)^{2}\right) U(\omega, s)=(A+B) s+\left(A K_{2}\left(\omega-\omega_{2}\right)^{2}+B K_{1}\left(\omega-\omega_{1}\right)^{2}\right)$

where $A$ and $B$ can be determined by using boundary conditions. From (A-6), it is straightforward to verify that equation (A-6) in Fourier-Laplace domain has the general form of $\operatorname{PDE}(7)$ in space-time domain.

\section{Appendix B}

\section{Proof for theorem 1:}

In order to find the propagator of a linear case of equation (10), we need to consider the isotropic case for PDE (10) where $K=K_{1}=K_{2}$ is constant, i.e.:

$$
\frac{\partial^{2} u}{\partial t^{2}}=K \frac{\partial}{\partial t}\left(\left(\frac{\partial}{\partial x}-j \omega_{1}\right)^{2}+\left(\frac{\partial}{\partial x}-j \omega_{2}\right)^{2}\right) u-K^{2}\left(\frac{\partial}{\partial x}-j \omega_{1}\right)^{2}\left(\frac{\partial}{\partial x}-j \omega_{2}\right)^{2} u
$$

To find the propagator of PDE (B-1), let us consider the following initial conditions for this equation:

$$
\begin{aligned}
& u(x, 0)=\delta(x) \\
& \frac{\partial u(x, 0)}{\partial t}=0
\end{aligned}
$$


where $\delta(x)$ is a Dirac delta function. By taking the Laplace transform from the both sides of equation (A-1), this equation is written as:

$$
\begin{aligned}
& s^{2} U(x, s)-s u(x, 0)-\frac{\partial u(x, 0)}{\partial t}=s K\left(\left(\frac{\partial}{\partial x}-j \omega_{1}\right)^{2}+\left(\frac{\partial}{\partial x}-j \omega_{2}\right)^{2}\right) U(x, s) \\
& -K\left(\left(\frac{\partial}{\partial x}-j \omega_{1}\right)^{2}+\left(\frac{\partial}{\partial x}-j \omega_{2}\right)^{2}\right) u(x, 0)-K^{2}\left(\frac{\partial}{\partial x}-j \omega_{1}\right)^{2}\left(\frac{\partial}{\partial x}-j \omega_{2}\right)^{2} U(x, s)
\end{aligned}
$$

where $U(x, s)$ is the Laplace transform of $u(x, t)$. By using equations (B-2) and (B-3) in equation (B-4) and taking the Fourier transform from the both sides of equation (B-4), one can obtain:

$$
\begin{aligned}
& s^{2} U(\omega, s)-s=s K\left(\left(j \omega-j \omega_{1}\right)^{2}+\left(j \omega-j \omega_{2}\right)^{2}\right) U(\omega, s)-K\left(\left(j \omega-j \omega_{1}\right)^{2}+\left(j \omega-j \omega_{2}\right)^{2}\right) \\
& -K^{2}\left(j \omega-j \omega_{1}\right)^{2}\left(j \omega-j \omega_{2}\right)^{2} U(\omega, s)
\end{aligned}
$$

where $U(\omega, s)$ is the Fourier transform of $U(x, s)$. From equation (B-5), $U(\omega, s)$ is calculated as:

$$
U(\omega, s)=\frac{s+K\left(\omega-\omega_{1}\right)^{2}+K\left(\omega-\omega_{2}\right)^{2}}{\left(s+K\left(\omega-\omega_{1}\right)^{2}\right)\left(s+K\left(\omega-\omega_{2}\right)^{2}\right)}
$$

By taking the inverse Laplace transform from the both sides of equation (B-6), one can conclude:

$$
U(\omega, t)=\frac{\left(\omega-\omega_{2}\right)^{2} \exp \left(-K\left(\omega-\omega_{1}\right)^{2} t\right)-\left(\omega-\omega_{1}\right)^{2} \exp \left(-K\left(\omega-\omega_{2}\right)^{2} t\right)}{\left(\omega-\omega_{2}\right)^{2}-\left(\omega-\omega_{1}\right)^{2}}
$$

Or

$$
U(\omega, t)=\left(\frac{\omega}{2\left(\omega_{1}-\omega_{2}\right)}+\frac{\left(\omega_{1}-3 \omega_{2}\right)}{4\left(\omega_{1}-\omega_{2}\right)}+\frac{\left(\omega_{1}-3 \omega_{2}\right)\left(\omega_{1}+\omega_{2}\right)+4 \omega_{2}^{2}}{8\left(\omega_{1}-\omega_{2}\right)\left(\omega-\frac{\omega_{1}+\omega_{2}}{2}\right)}\right) \exp \left(-K\left(\omega-\omega_{1}\right)^{2} t\right)
$$




$$
+\left(\frac{\omega}{2\left(\omega_{2}-\omega_{1}\right)}+\frac{\left(\omega_{2}-3 \omega_{1}\right)}{4\left(\omega_{2}-\omega_{1}\right)}+\frac{\left(\omega_{2}-3 \omega_{1}\right)\left(\omega_{1}+\omega_{2}\right)+4 \omega_{1}^{2}}{8\left(\omega_{2}-\omega_{1}\right)\left(\omega-\frac{\omega_{1}+\omega_{2}}{2}\right)}\right) \exp \left(-K\left(\omega-\omega_{2}\right)^{2} t\right)
$$

Finally by taking the inverse Fourier transform of the both sides of equation $(\mathrm{B}-8), u(x, t)$ is calculated as equation (11).

\section{REFERENCES}

[1] K. Max Wong, J. Wu, T.N. Davidson, Q. Jin, and P.C. Ching, "Performance of Wavelet PacketDivision Multiplexing in Impulsive and Gaussian Noise", IEEE Transactions on Communications, Vol. 48, No. 7, pp. 1083-1086, 2000.

[2] P. Banelli, and S. Cacopardi, "Theoretical Analysis and Performance of OFDM Signals in Nonlinear AWGN Channels", IEEE Transactions on Communications, Vol. 48, No. 3, pp. 430$441,2000$.

[3] P. Perona, and J. Malik, "Scale-Space and Edge Detection using Anisotropic Diffusion", IEEE Transactions on Pattern Recognition and Machine Intelligence, Vol. 12, No. 7, pp. 629-639, 1990.

[4] G. Gerig, O. Kubler, R. Kikinis, and F. Jolesz, "Nonlinear Anisotropic Filtering of MRI Data", IEEE Transactions on Medical Imaging, Vol. 11, No. 2, pp. 221-232, 1992.

[5] G. Sapiro, D.L. Ringach, "Anisotropic Diffusion of Multi-Valued Images with Applications to Color Filtering”, IEEE Transactions on Image Processing, Vol. 5, No. 11, pp. 1582-1586, 1996.

[6] M.J. Black, G. Sapiro, D.H. Marimont, and D. Heeger, "Robust Anisotropic Diffusion", IEEE Transactions on Image Processing, Vol.. 7, No.3, pp. 421-432, 1998.

[7] D. Tschumperie, "Fast Anisotropic Smoothing of Multi-Valued Images using CurvaturePreserving PDEs", International Journal of Computer Vision, Vol. 86, No. 1, pp. 65-82, 2006

[8] S. Mahmoodi, "Anisotropic Diffusion for Noise Removal of Band Pass Signals", Signal Processing, Vol. 91, No. 5, pp. 1298-1307, 2011. 
[9] S. Mahmoodi, "Discontinuity Preserving Noise Removal Method based on Anisotropic Diffusion for Band Pass Signals", IEEE International Workshop on Multi-Media Signal Processing, pp. 362-366, 2013.

[10] H. Harvin, and B. Joricke, The Uncertainty Principle in Harmonic Analysis, Springer-Verlag, 1994.

[11] C.A. Palma, F.A.M. Cappabianco, J.S. Ide, P.A.V. Miranda, "Anisotropic Diffusion Operation and Limitations- Magnetic Resonance Evaluation", The International Federation of Automatic Control, Cape Town, South Africa, pp. 3887-3892, 2014. 


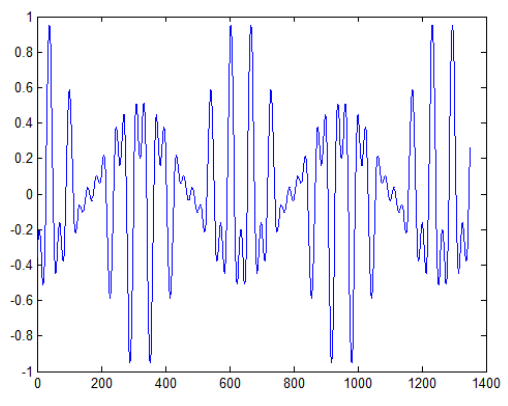

$a$

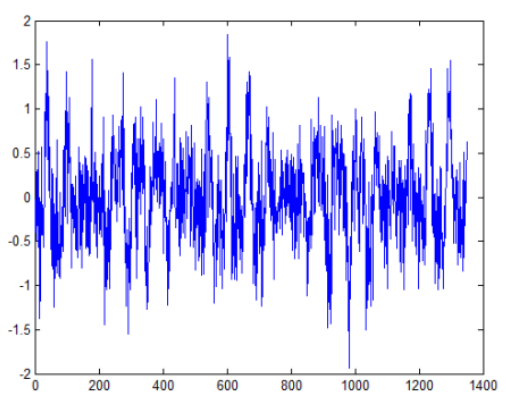

$b$

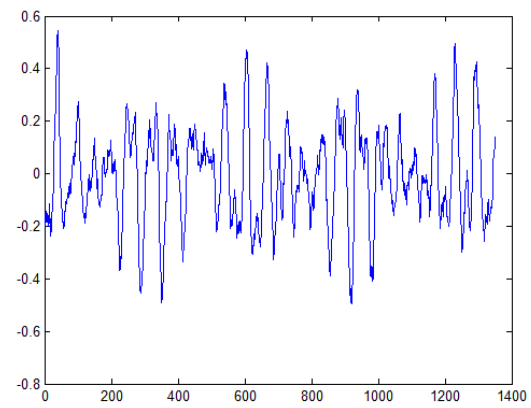

Figure (1): Noise reduction in a signal with two carrier signals and no discontinuity a) noiseless signal with two carrier signals with frequencies $\omega_{1}=0.1, \omega_{2}=0.2 \mathrm{rad} / \mathrm{s}$ and with no discontinuity b) Noisy signal with white zero mean Gaussian noise with $\mathrm{SNR}_{\mathrm{dB}}=-0.82 \mathrm{c}$ ) the smoothed signal with $K=1, t=53$ and filter size $=150$
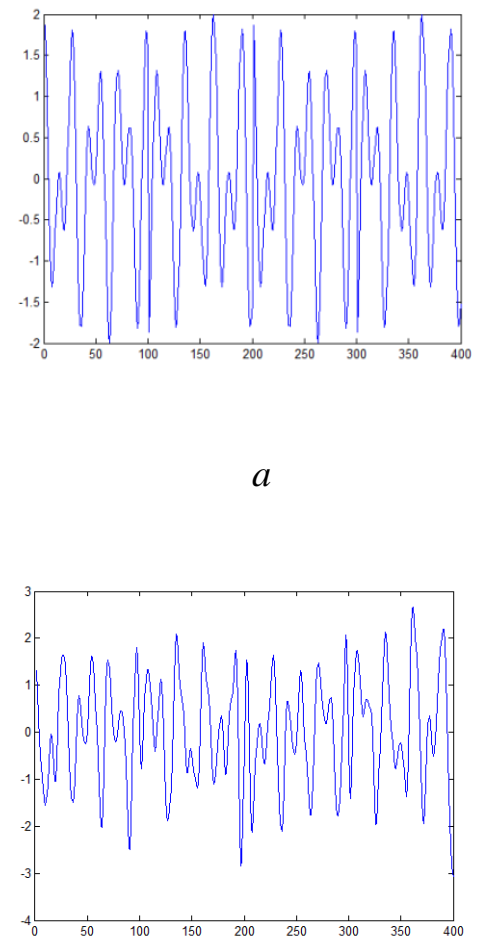

$c$

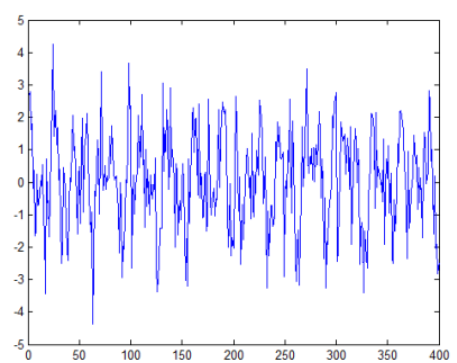

$b$

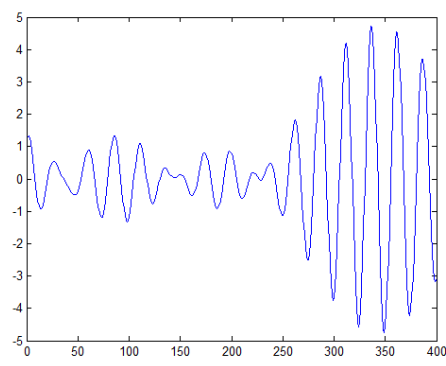

$d$

Figure 2: Gaussian noise removal of a signal modulated with two carrier signals with frequencies 0.25 and $0.45 \mathrm{rad} / \mathrm{s}$ a) original noiseless signal containing three discontinuities at locations 100,200 and $300 \mathrm{~b})$ the signal of figure (2-a) contaminated with Gaussian noise $\left.\left(\mathrm{SNR}_{\mathrm{dB}}=-0.19\right) \mathrm{c}\right)$ the signal smoothed by the double carrier anisotropic diffusion with $q=10$ and $\Delta t=0.01$ after 600 iterations d) the signal smoothed by the single frequency anisotropic diffusion [8] with a frequency of 0.25 and similar parameters 


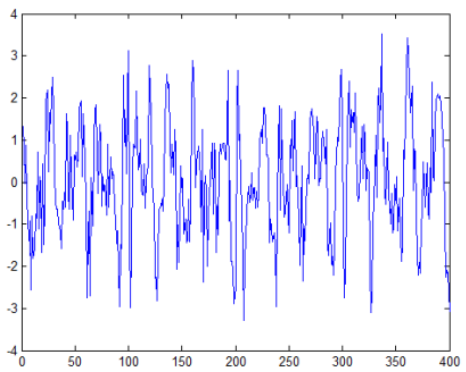

$a$

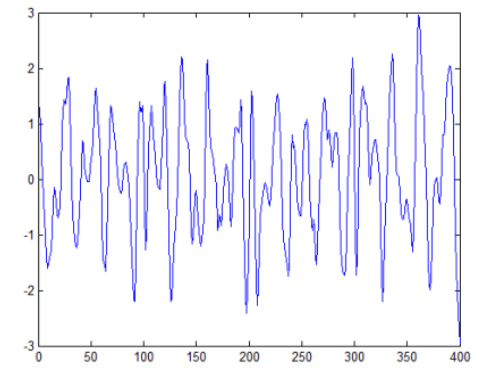

$b$

Figure (3): The noisy signal of figure (2-b) smoothed by using the explicit method for the double carrier anisotropic diffusion after a) 1000 iterations and b) 4000 iterations

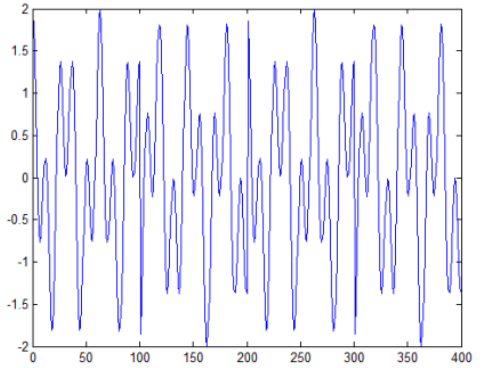

$a$

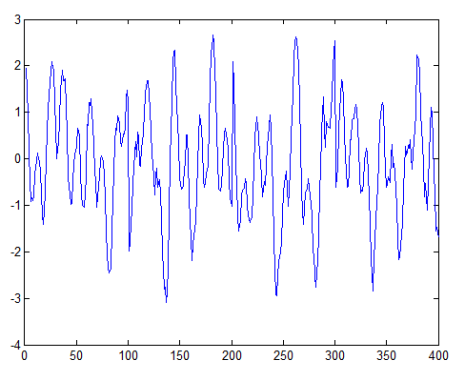

$b$

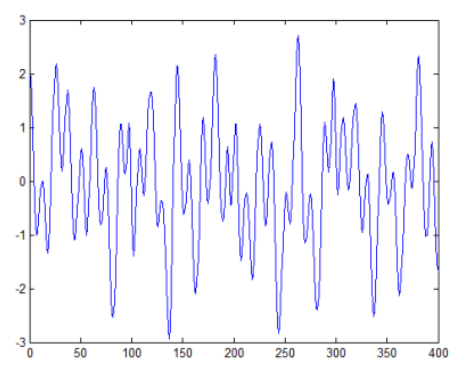

$c$

Figure (4): Smoothing a signal contaminated with correlated Gaussian noise a) Original noiseless signal with discontinuities at locations 100,200 , and 300 b) Noisy signal with $S N R_{d B}=5.44$ contaminated with correlated signal c) Smoothed signal by using our proposed algorithm with $\Delta t=0.01$ and $q=10$, after 600 iterations

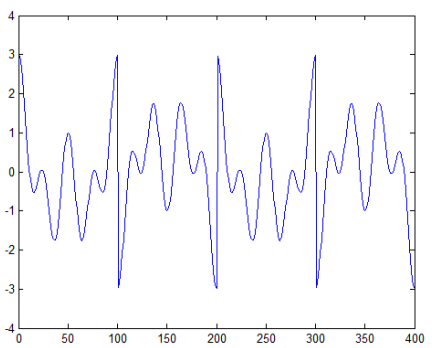

$a$

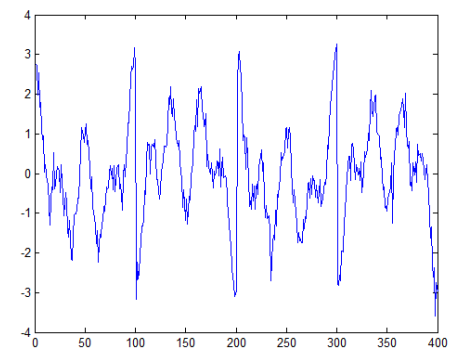

$b$

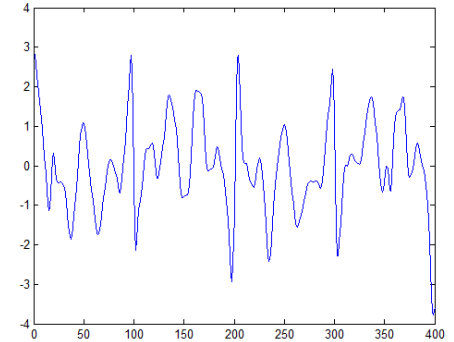

$c$

Figure 5: signal smoothing for OFDM signals with three carrier frequencies a) Noiseless signal with three carrier signals $\left(\omega_{1}=0.0625, \omega_{2}=0.125\right.$ and $\left.\omega_{3}=0.25 \mathrm{rad} / \mathrm{s}\right)$ with discontinuities at locations 100, 200, and $300 \mathrm{~b}$ ) White Gaussian noise is added to the signal of figure (5-a) to produce a noisy signal with $S N R_{d B}=12.04 \mathrm{c}$ ) Smoothed signal by using our algorithm with $\Delta t=0.01$ and $q=10$ after 400 iterations 

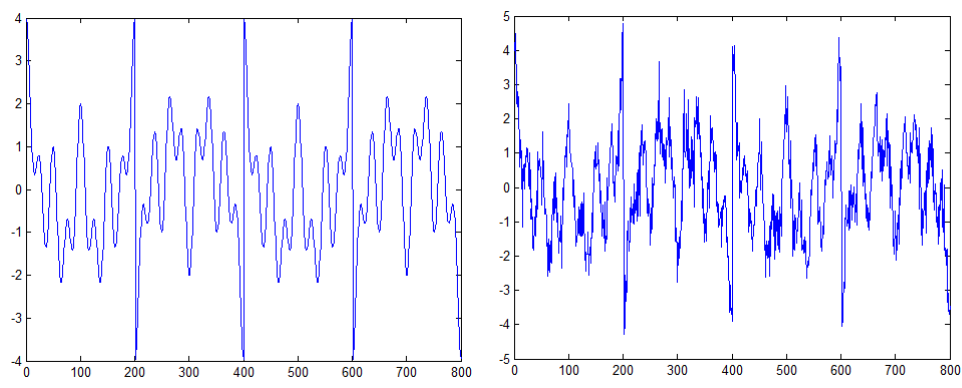

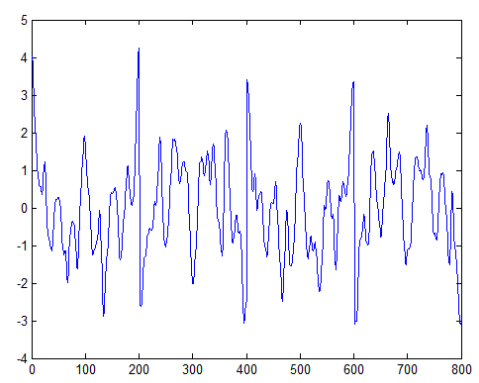

C

Figure 6: signal smoothing for OFDM signals with four carrier frequencies a) Noiseless signal with four carrier signals $\left(\omega_{1}=0.0313, \omega_{2}=0.0625, \omega_{3}=0.125\right.$ and $\left.\omega_{4}=0.25 \mathrm{rad} / \mathrm{s}\right)$ with discontinuities at locations 200, 400, and $600 \mathrm{~b}$ ) white Gaussian noise is added to the signal of figure (6-a) to produce a noisy signal with $S N R_{d B}=8.92 \mathrm{c}$ ) Smoothed signal by using our algorithm with $\Delta t=0.01$ and $q=10$ after 600 iterations

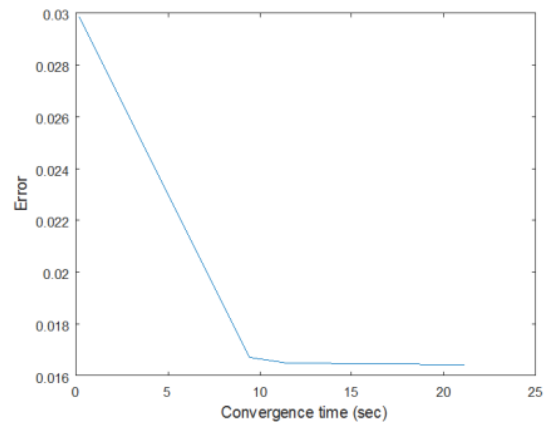

Figure (7): The plot describing the relationship between the error term (the difference between noiseless and filtered signals) and the convergence time

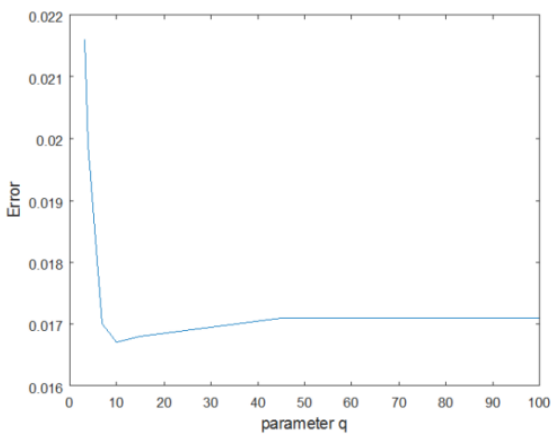

$a$

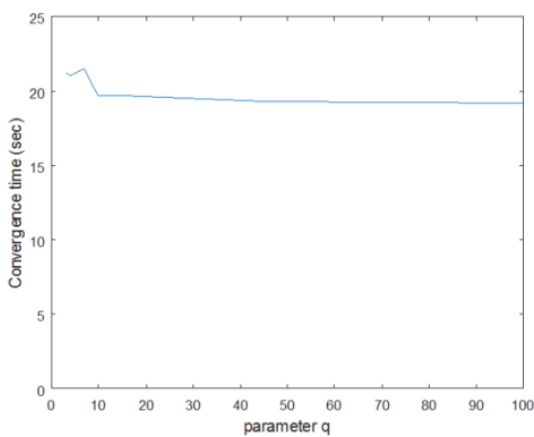

$b$

Figure (8): a) The error term and b) the convergence time of the anisotropic diffusion algorithm with double carrier frequencies $\left(\omega_{1}=0.125, \omega_{2}=0.5\right)$ with respect to $q$ for $\Delta t=0.01$ and $S N R_{d B}=4.38$ 


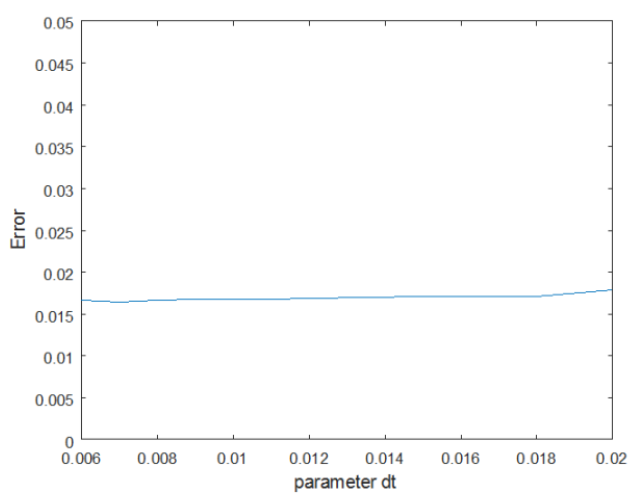

$a$

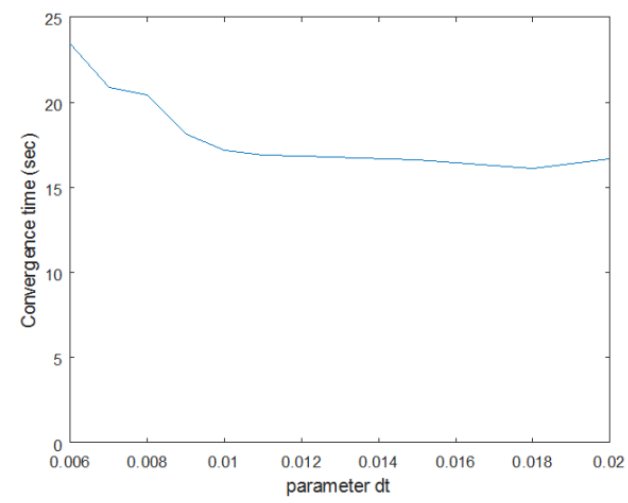

$b$

Figure (9): a) The error term and b) the convergence time of the anisotropic diffusion algorithm with double carrier frequencies $\left(\omega_{1}=0.125, \omega_{2}=0.5\right)$ with respect to $\Delta t$ for $q=10$ and $S N R_{d B}=4.38$

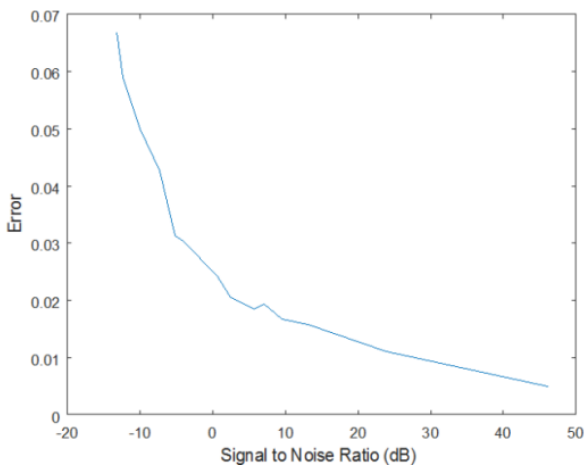

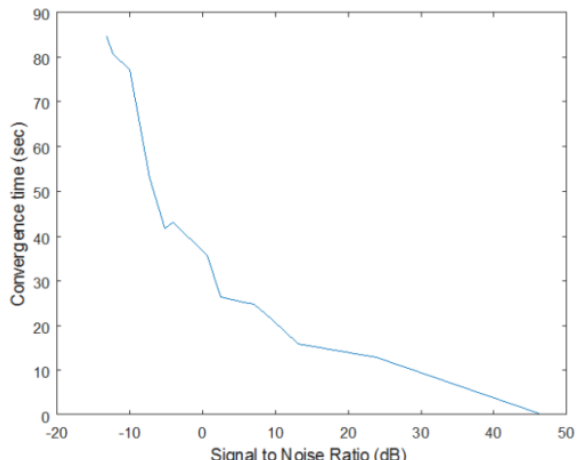

$b$

Figure (10): a) The error term and b) the convergence time of the anisotropic diffusion algorithm with double carrier frequencies $\left(\omega_{1}=0.125, \omega_{2}=0.5\right)$ with respect to $S N R_{d B}$ of the noisy signal for $\Delta t=0.01$ and $q=10$.

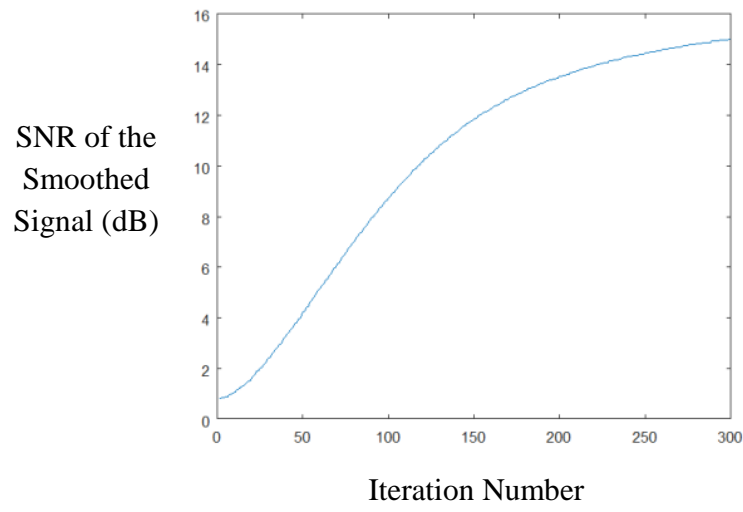

Figure (11): The evolution of SNR (SNR of the smoothed signal in each iteration) with respect to the iteration numbers for the anisotropic diffusion with double carrier frequencies contaminated with the Gaussian noise for $q=10$ and $\Delta t=0.01$ 